\title{
Detecting hotspots of atmosphere-vegetation interaction via slowing down - Part 2: Application to a global climate model
}

\author{
S. Bathiany ${ }^{1}$, M. Claussen ${ }^{1,2}$, and K. Fraedrich ${ }^{1,2}$ \\ ${ }^{1}$ Max Planck Institute for Meteorology, KlimaCampus Hamburg, Germany \\ ${ }^{2}$ Meteorologisches Institut, Universität Hamburg, KlimaCampus Hamburg, Germany \\ Correspondence to: S. Bathiany (sebastian.bathiany@zmaw.de)
}

Received: 27 June 2012 - Published in Earth Syst. Dynam. Discuss.: 20 July 2012

Revised: 8 December 2012 - Accepted: 5 February 201 - Published: 26 February 2013

\begin{abstract}
Early warning signals (EWS) have become a popular statistical tool to infer stability properties of the climate system. In Part 1 of this two-part paper we have presented a diagnostic method to find the hotspot of a sudden transition as opposed to regions that experience an externally induced tipping as a mere response. Here, we apply our method to the atmosphere-vegetation model PlanetSimulator (PlaSim) - VECODE using a regression model. For each of two vegetation collapses in PlaSim-VECODE, we identify a hotspot of one particular grid cell. We demonstrate with additional experiments that the detected hotspots are indeed a particularly sensitive region in the model and give a physical explanation for these results. The method can thus provide information on the causality of sudden transitions and may help to improve the knowledge on the vulnerability of certain subsystems in climate models.
\end{abstract}

\section{Introduction}

The phenomenon of slowing down, an increase in a system's relaxation time resulting from a loss in stability, has been studied for a long time in various systems (for example Collins and Teh, 1973; Wissel, 1984; Wolff, 1990). The effect is often illustrated with the prototype example of a one-dimensional potential whose shape determines the system's deterministic dynamics, and a ball which characterises its state (Fraedrich, 1979; Scheffer et al., 2001). The deterministic system is supposed to approach an equilibrium (in terms of dynamical systems, a stable fixed point), determined by a minimum in the potential. If by varying an external control parameter the potential becomes flatter, the return time to the equilibrium increases and the eigenvalue as determined by a linear stability analysis increases towards 0 (in a time-continuous system).

As the underlying dynamics are rarely known in complex systems, it has been suggested to infer stability changes of a current equilibrium from statistical indicators. If the system's state is subject to external random perturbations (usually assumed as white noise of constant noise level), a loss in stability will lead to an increase in autocorrelation and (at least if the noise is additive) variance. These changes can be measured if the change in external conditions is slow enough to allow a sufficiently precise sampling of the statistical indicators. Assuming that the existence of a non-linear threshold, in the extreme case a catastrophic bifurcation point, is known, then an attempt can be made to predict when a sudden transition will occur (Thompson and Sieber, 2011a,b). For this reason, statistical indicators of slowing down have been referred to as early warning signals (EWS; Scheffer et al., 2009).

The generality of the concept has recently inspired the search for slowing down and EWS in various contexts such as ecological models (Carpenter and Brock, 2006; van Nes and Scheffer, 2007; Guttal and Jayaprakash, 2008; Contamin and Ellison, 2009), living populations in laboratories (Drake and Griffen, 2010; Veraart et al., 2012) and real ecosystems (Carpenter et al., 2011), geological climate records (Dakos et al., 2008; Ditlevsen and Johnsen, 2010), and climate models (Kleinen et al., 2003; Held and Kleinen, 2004; Livina and Lenton, 2007; Lenton et al., 2009; Lenton, 2011).

However, most of these studies address the problem within the one-dimensional framework explained above and thereby considerably reduce the spatial complexity of real systems. Palaeoclimate records are inherently one-dimensional and 
generally yield spatially integrated information. In ocean circulation models, integrated mass fluxes are often a useful quantity to characterise large-scale changes. For example, Held and Kleinen (2004) study a collapse of the meridional overturning circulation and obtain a single time series by projecting on the critical mode of the transition ("degenerate fingerprinting").

EWS in ecological systems have recently been studied in a spatially more explicit way (Oborny et al., 2005; Guttal and Jayaprakash, 2009; Donangelo et al., 2010; Dakos et al., 2010, 2011). However, the latter studies involve two simplifications: first, the analysed systems involve interactions which couple grid cells in a spatially homogeneous way. Second, the grid is constructed from identical elements with individual tipping points and the system's boundaries are well defined.

In this regard, the interactions between terrestrial ecosystems and the atmosphere pose a more difficult problem. Considering a global climate model, all land cells are globally coupled via the atmosphere, the spatial coupling is inhomogeneous, and the critical region producing a tipping point is embedded in a larger system with other dynamical characteristics.

In such a complex setting, it is of interest not only if or when a tipping occurs, but also where it occurs and causally originates (hotspot). In Part 1 of this article (Bathiany et al., 2013) we have shown that the detection of local EWS at individual grid cells is generally not sufficient to solve this problem. However, the hotspot as a nucleus of the abrupt transition can potentially be identified with a degenerate fingerprinting approach by determining the area which maximises an EWS. Here, we apply the method to Holocene vegetation dynamics in North Africa and Southwest Asia in the atmosphere-vegetation model PlanetSimulator (PlaSim) - VECODE. North Africa is a region where atmospherevegetation interactions have been particularly important during the Holocene (Claussen, 1998). In a number of climate models rapid transitions due to strong feedbacks (Claussen et al., 1999; Renssen et al., 2003) and multiple equilibria (Claussen, 1994, 1997, 1998; Zeng and Neelin, 2000; Wang and Eltahir, 2000; Irizarry-Ortiz et al., 2003) have been found.

In Sect. 2, we briefly introduce the two models, the methods of coupling, as well as the dynamic vegetation changes simulated by PlaSim-VECODE. In Sect. 3 we discuss the restrictions of applying EWS to time series generated by PlaSim-VECODE, introduce a regression model, and derive parameter values to match our PlaSim-VECODE results. We then apply the hotspot detection scheme to our regression model in Sect. 4. In Sect. 5, we verify the results with PlaSim-VECODE and give a physical explanation of the model's behaviour. Section 6 provides our conclusions.

\section{Mid-Holocene vegetation dynamics in PlaSim-VECODE}

To simulate mid-Holocene vegetation dynamics, we couple the atmosphere model Planet Simulator (PlaSim; Fraedrich et al., 2005; Fraedrich, 2012) to the simple dynamic global vegetation model VECODE (Brovkin et al., 1997, 2002). The experimental setup is identical to Bathiany et al. (2012): sea surface temperatures are prescribed from present-day observations, atmospheric $\mathrm{CO}_{2}$ is fixed at $280 \mathrm{ppm}$, and the resolution is T21 with 10 vertical layers. Equilibrium vegetation cover $V^{*}$ in VECODE directly depends on annual precipitation $P$ :

$V^{*}= \begin{cases}0 & \text { if } P<P_{1} \\ 1 & \text { if } P>P_{2} \\ 1.03-\frac{1.03}{1+\alpha\left(\frac{P-P_{1}}{\exp (\gamma \delta)}\right)^{2}} & \text { otherwise, }\end{cases}$

with

$P_{1}=\beta \exp (\gamma \delta / 2)$

$P_{2}=\beta \exp (\gamma \delta / 2)+\frac{\exp (\gamma \delta)}{\sqrt{0.03 \alpha}}$.

In order to allow for vegetation collapses, we implement a steeper threshold in the equilibrium vegetation cover's response to precipitation by choosing $\alpha=0.0011, \beta=140$, $\gamma=1.7 \times 10^{-5}$, and $\delta=\mathrm{GDD}_{0}-900 \mathrm{~K}$, where $\mathrm{GDD}_{0}$ are the growing degree days above $0^{\circ} \mathrm{C}$ (a function of temperature only). This version is called the modified VECODE in Bathiany et al. (2012) (Fig. 1). VECODE distinguishes trees and grass as the only vegetation types. The surface cover types, trees, grass and desert, have different physical properties which are constant over time.

PlaSim and VECODE can be coupled in two ways: in a transient mode (PlaSim-VECODE-tr), we use an annual coupling, and vegetation cover fractions at each grid box approach their equilibrium according to a linear relaxation law using a climate dependent timescale. In equilibrium mode (PlaSim-VECODE-eq), we iteratively run PlaSim with fixed vegetation cover for several years, and then set vegetation cover to its new equilibrium corresponding to the multi-year average of $P$ and $\mathrm{GDD}_{0}$. This mode thus corresponds to an asynchronous coupling.

The asynchronous coupling corresponds to the limit of infinitely fast vegetation dynamics so that there is no timescale separation between $P$ and $V$ anymore. In addition, the variability of $P$ (the fast subsystem) is averaged out and replaced by its mean response. This way, the stable deterministic equilibria of the slow part of the system can be identified because the system is always very close to these equilibria. In this sense, the interactive timescale of several years in PlaSimVECODE-tr and the large variability provide a more realistic case. However, the relationship of climate and vegetation timescale in the model is an empirical fit to observations 


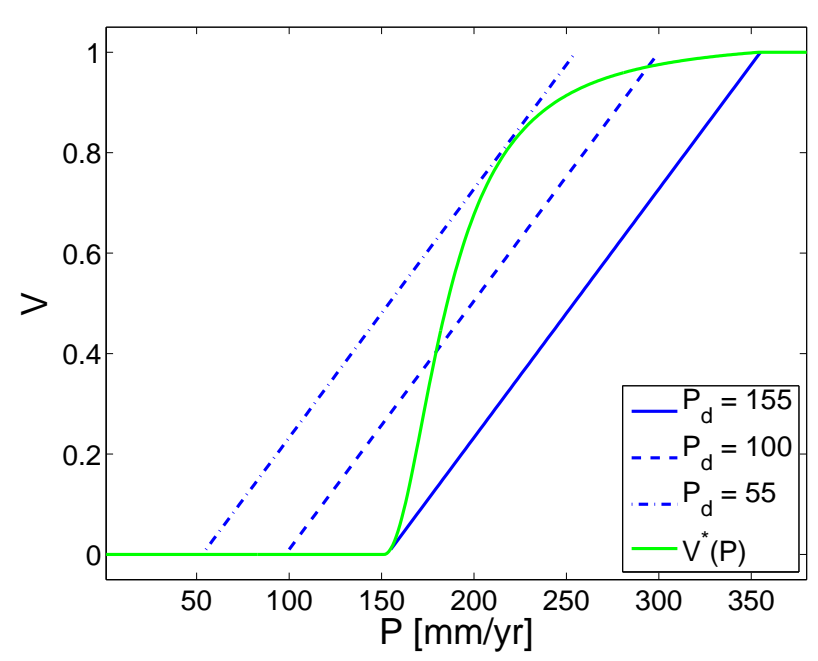

Fig. 1. Conceptual stability diagram to illustrate multiple equilibria in the atmosphere-vegetation system. $P$ : annual precipitation, $V$ : vegetation cover fraction. Green line: equilibrium vegetation cover $V^{*}(P)$ as in the modified VECODE (Eq. 1) with $\delta=9100$. Blue lines: dependency of $P$ on $V$, here assumed to be linear. As orbital forcing causes background precipitation $P_{\mathrm{d}}$ to decrease, the system reaches a bifurcation point (here at approx. $P_{\mathrm{d}}=55$ where the green equilibrium disappears and the system would have to fall into the remaining desert state).

from different ecosystems that may not be directly transferable to changes in time at one and the same location. As it is our aim to investigate the stability properties of PlaSimVECODE rather than its realism, we accept this limitation as a side-effect of the model's simplicity.

When running PlaSim-VECODE-tr with orbital forcing from $9 \mathrm{k}$ ( $\mathrm{k}$ is kiloyears before present) to $2 \mathrm{k}$, we obtain two vegetation collapses in different regions at different times (Bathiany et al., 2012). The spatial and temporal features of these transitions are presented in Figs. 2 and 3. From $9 \mathrm{k}$ to $6 \mathrm{k}$, almost all land cells are at least partly covered by vegetation and cover fractions show large fluctuations due to natural climate variability but almost no trend. Around $5.5 \mathrm{k}$, vegetation cover in large parts of northern Africa and southwestern Asia collapses and thereafter stays in desert-like conditions. Interestingly, the timing of this collapse corresponds to palaeoclimate time series from a sediment core (deMenocal et al., 2000) and earlier model studies (Claussen et al., 1999), despite differences in the models and our modifications to the vegetation model. Around $3.5 \mathrm{k}$, a similar abrupt event occurs in a more confined region in the Sahel region. In the following, we refer to these two sudden transitions as collapse 1 ( $5.5 \mathrm{k}$ event) and collapse 2 ( $3.5 \mathrm{k}$ event).

The two vegetation collapses are related to the large atmosphere-vegetation feedback in the model which can allow for multiple equilibria. In PlaSim-VECODE-eq, multiple steady states in the region of collapse 1 can be found until approx. $7 \mathrm{k}$., and in the region of collapse 2 at $4.5 \mathrm{k}-5 \mathrm{k}$ (see
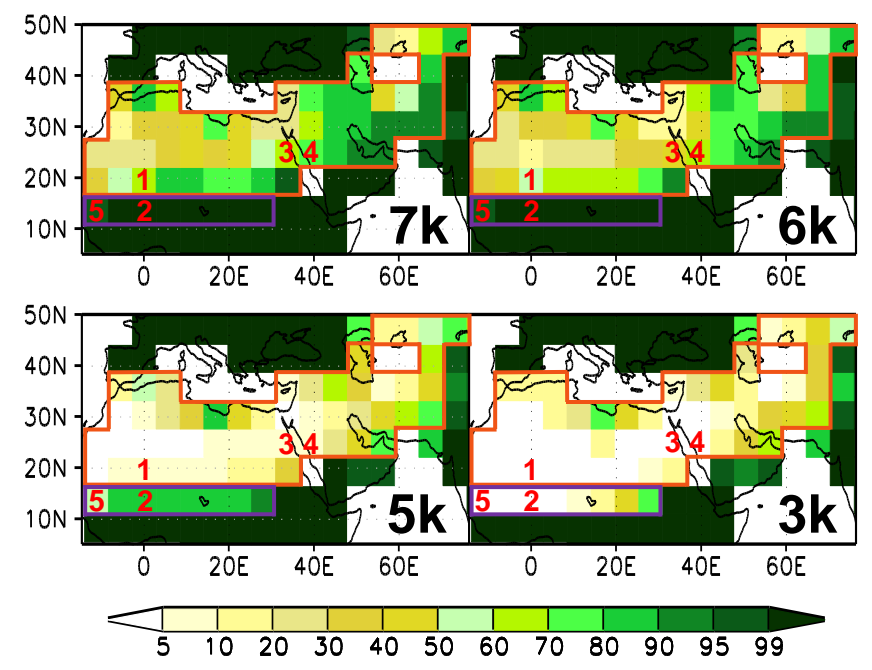

Fig. 2. Mean vegetation cover fractions (trees + grass) in $\%$ in the transient PlaSim-VECODE experiment from $9 \mathrm{k}$ to $2 \mathrm{k}$. Vegetation cover is averaged over $200 \mathrm{yr}$ starting from the indicated year $(7 \mathrm{k}$, $6 \mathrm{k}, 5 \mathrm{k}$, and $3 \mathrm{k}$, respectively). Numbers 1 to 5 denote the individual grid cells referred to in the text. The red region encloses the 52 grid cells considered in RM1, the purple region encloses the 8 grid cells considered in RM2.

Figs. 6, 7 and Table 2 in Bathiany et al., 2012). For each of these orbital forcings, starting from a forest world leads to a partly vegetated state (in the following called the "green equilibrium"), while starting from desert conditions leads to a dry state ("desert equilibrium") in PlaSim-VECODEeq. However, due to the large climate variability and nonlinearities in the model formulation, a noise-induced transition (Horsthemke and Lefever, 1984) can occur and multiple steady states are not found in PlaSim-VECODE-tr, where natural variability is large. The collapses presented in Figs. 2 and 3, although a result of the intrinsic multiple equilibria in the system, thus do not exactly coincide with the deterministic bifurcation points, but rather result from a sudden change in the system's probability density function.

\section{A stochastic model for EWS analysis}

\subsection{Idealised model setup}

To further analyse the stability properties of the modified PlaSim-VECODE and to find hotspots in the model, we apply our hotspot detection method.

Following our algorithm for hotspot detection presented in Part 1, we first generate a number of time slices for fixed orbital forcing. To analyse collapse 1 , we choose orbital forcings corresponding to $9 \mathrm{k}, 8.5 \mathrm{k}, 8 \mathrm{k}, 7.5 \mathrm{k}, 7 \mathrm{k}, 6.5 \mathrm{k}$ and $6 \mathrm{k}$ (dashed red lines in Fig. 3, top); to analyse collapse 2 we choose $5.5 \mathrm{k}, 5 \mathrm{k}, 4.5 \mathrm{k}, 4 \mathrm{k}$, and $3.6 \mathrm{k}$ (dashed red lines in Fig. 3, bottom). As any year is associated with a particular orbital forcing we refer to this forcing as an orbit year. Each 

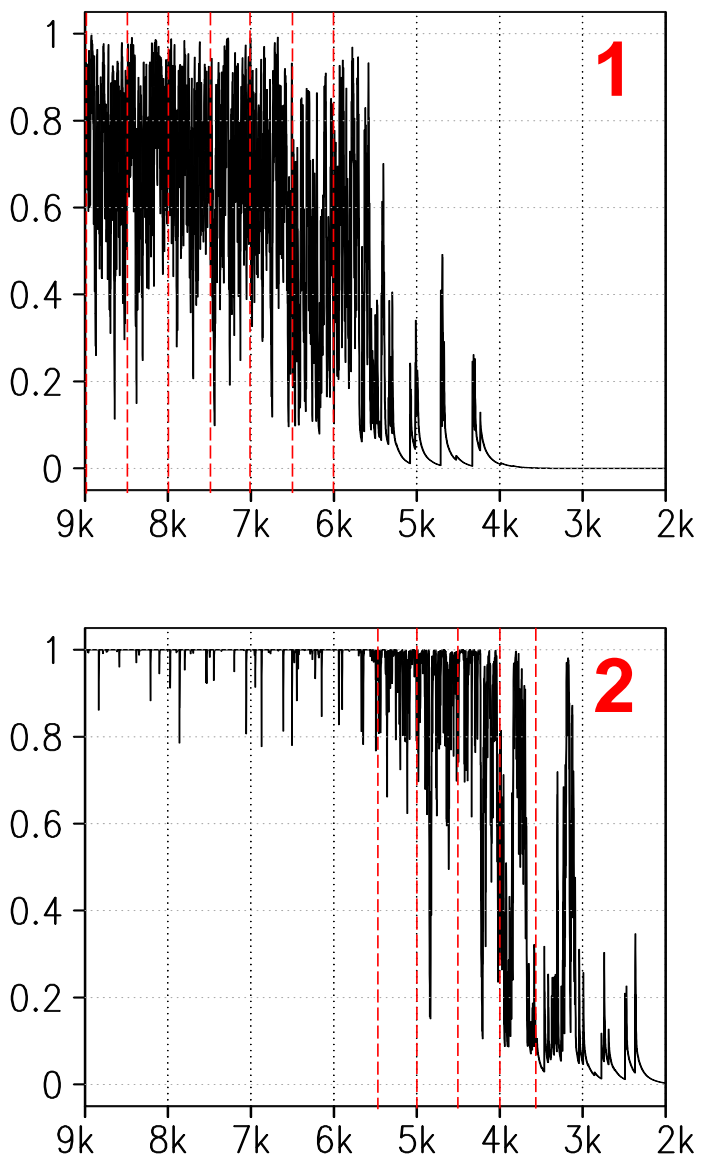

Fig. 3. Vegetation cover fraction in the transient PlaSim-VECODE experiment from $9 \mathrm{k}$ to $2 \mathrm{k}$. Two single grid cells are shown, indicated as grid cells 1 and 2 in Fig. 2. The vertical dashed lines indicate the $B$ values of the $20000 \mathrm{yr}$ long stationary PlaSimVECODE-tr simulations used to construct RM1 (top) and RM2 (bottom).

time slice simulation consists of $20000 \mathrm{yr}$ in transient coupling mode.

However, a direct application of the hotspot detection scheme to these time series is not adequate for the following three reasons:

1. Due to the distinction of cases in Eq. (1), vegetation cover fraction $V$ does not always show free variations but is often exactly 0 or 1 . The application of EWS is not suited for such a case as the stability properties of the equilibrium cannot be sampled properly. For example, before reaching a desert state, the vegetation cover fraction shows an exponential decay after a particularly wet year and stays constant afterwards (Fig. 3). More importantly, the same phenomenon occurs at the other limit of phase space, $V=1$. EWS like autocorrelation or variance then depend on the frequency of such cutoff events, which are not related to the stability of a climate state.
2. The timescale $\tau$ of dynamic vegetation cover change in VECODE depends on the system's state and thus contaminates the signal of slowing down. In particular, $\tau$ is large for dry regimes and small for wet regimes (see Fig. 3 in Bathiany et al., 2012). When background precipitation is reduced, an increased timescale will be reflected in an increased autocorrelation. This statedependent slowing down is not necessarily related to any change in stability and thus distorts the signal.

3. Atmospheric variability in PlaSim-VECODE-tr is too large to justify the small noise approximation. As explained above, the two collapses cannot be expected to coincide with a vanishing eigenvalue because they result from non-linear interactions between the amplitude of the multiplicative noise and the system's state (Bathiany et al., 2012).

Insofar, the prerequisites for an application of EWS-based analysis are in conflict with the case of PlaSim-VECODE-tr. We therefore use the regression model introduced in Part 1 of our study. In this stochastic model, precipitation $P$ at any grid cell $i$ is described as a linear function of the vegetation cover fractions $V_{j}$ at all cells, following the concept of Brovkin et al. (1998), Wang (2004), and Liu et al. (2006):

$P_{i}^{t}=P_{0_{i}}+s_{i} B+\sum_{n=1}^{N} k_{i j} V_{j}^{t}+\sigma_{P} \eta_{i}^{t}$.

Vegetation dynamics are represented by the simple dynamic equation

$V_{i}^{t+1}=V_{i}^{t}+\frac{V_{i}^{*}\left(P_{i}^{t}\right)-V_{i}^{t}}{\tau}+\sigma_{V} \eta_{i}^{t}$,

with $t$ as the discrete time and a time step of one year. The bifurcation parameter $B$ is the time (in kiloyears before present) that corresponds to a certain orbital forcing. Decreasing $B$ implies an orbital forcing that evolves forward in time. This in turn tends to decrease $P$ during the late Holocene due to the impact of orbital forcing on Northern Hemisphere summer insolation and thus convective precipitation. Spatial interactions are captured by matrix $\mathbf{k}$, and climate variability is accounted for by a Gaussian white noise process $\eta_{i}$, which is also uncorrelated in space.

In contrast to PlaSim-VECODE, the three caveats listed above can be resolved within the framework of this regression model:

1. We remove the second condition in Eq. (1), thereby allowing for $V>1$ which corresponds to an extrapolation of the empirical $P(V)$ relation obtained in PlaSimVECODE. As a maximum of $V=1.03$ is still not exceeded, we accept the unphysical nature of cover fractions larger than 1 in the regression model.

2. We fix the dynamic timescale $\tau$ to a the climateindependent value of $5 \mathrm{yr}$ in agreement with Liu et al. (2006) and Bathiany et al. (2012). 
RM1
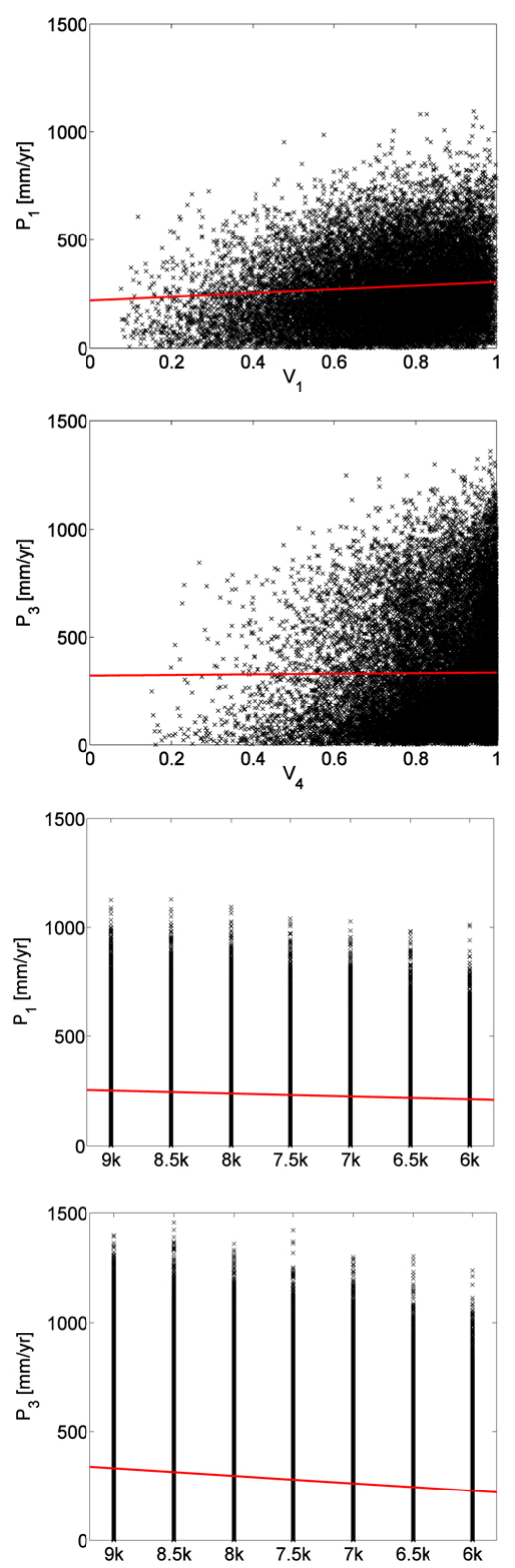

RM2
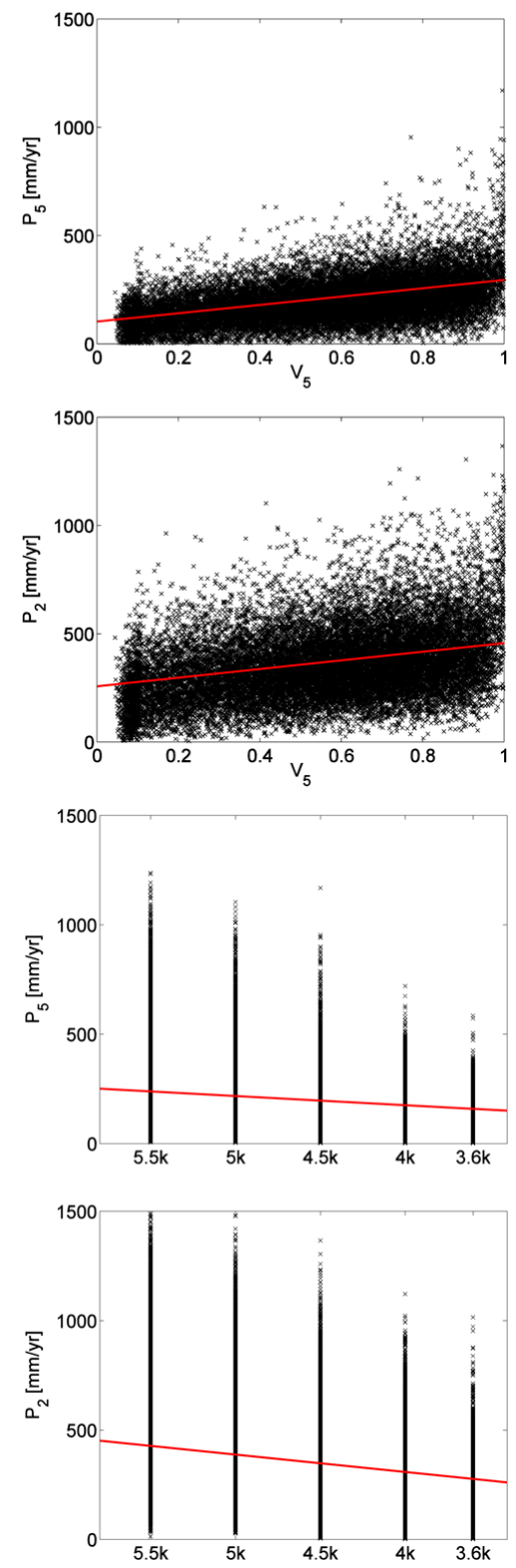

Fig. 4. Relation between precipitation $(P)$, vegetation cover fraction $(V)$ and orbital forcing from the stationary simulations with PlaSimVECODE-tr. The indices of $P$ and $V$ refer to the specific grid cells labelled in Fig. 2 (for example, $P_{3}$ vs. $V_{4}$ shows $P$ at cell 3 versus $V$ at cell 4 for all years and simulations). The four lower panels show the relation between $P$ and orbital forcing. Left: simulations used to derive RM1, right: simulations used to derive RM2. The slope of the red lines corresponds to parameters $k_{i j}$ and $s_{i}$ as obtained from the multiple regression, the intersects have been obtained by assuming mean conditions for all other predictor variables.

3. We prescribe a particularly small and constant noise level of $\sigma_{V}=0.00013$ with $\sigma_{P}=0$ (additive noise) or $\sigma_{P}=2$ with $\sigma_{V}=0$ (multiplicative noise).

We refer to this model as regression model 1 (RM1) when studying collapse 1, and as regression model 2 (RM2) when studying collapse 2 . Both models only differ in the number of grid cells and the parameter values.
To keep the regression models as simple as possible, we only include grid cells in northern Africa and southwestern Asia which show substantial fluctuations in vegetation cover. Grid cells with $V$ permanently close to 1 or 0 in all time slices are static elements of the system under consideration and can thus be interpreted as external conditions which are indirectly reflected in the constants $P_{0_{i}}$. For RM1, we include all grid cells where $V$ averaged over time and all time slices 

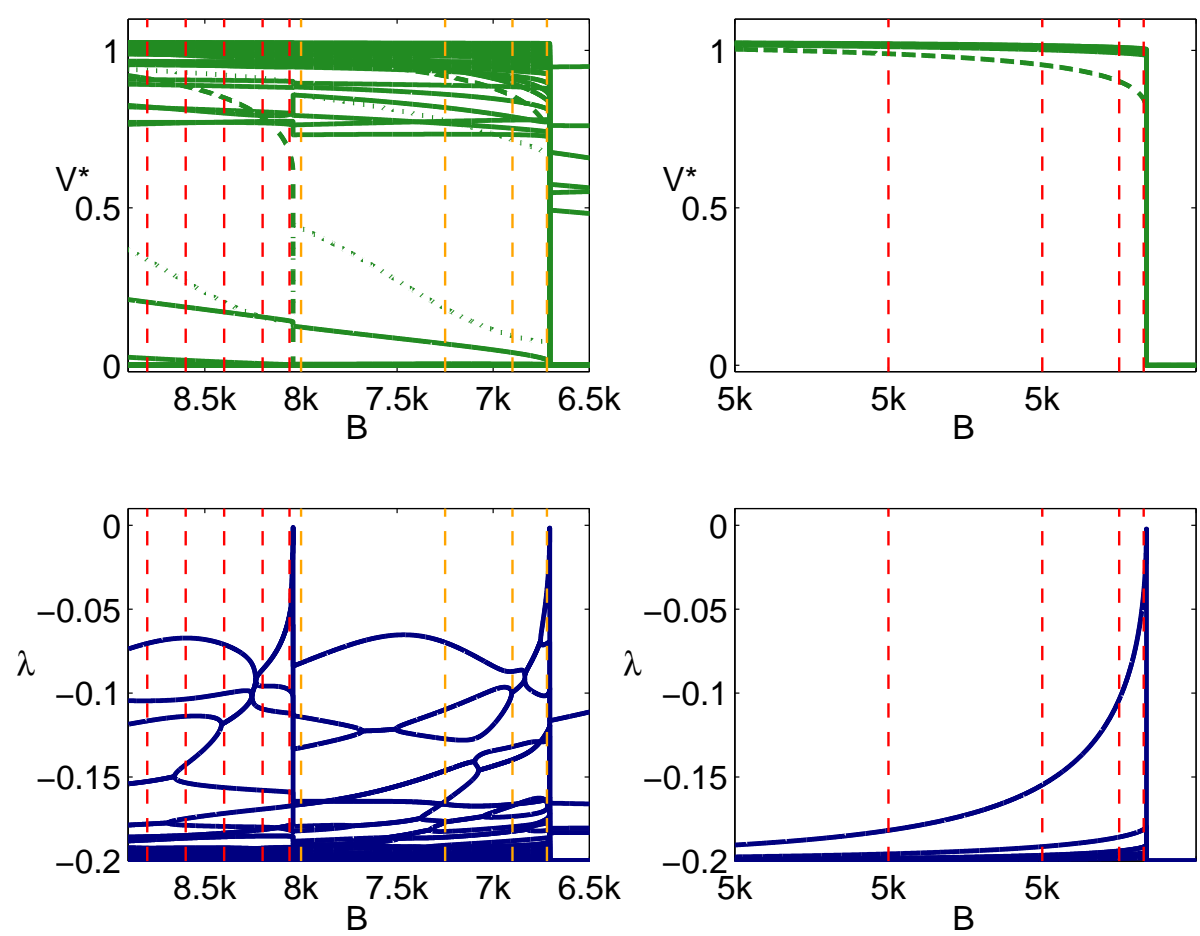

Fig. 5. Characteristics of RM1 (left) and RM2 (right), depending on parameter $B$. Top: Equilibrium vegetation cover at all elements (greenest solution). The elements identified as hotspots are dashed. Some elements are dotted only to be better distinguishable from others. Bottom: real part of eigenvalues characterising the linear stability of the corresponding solution of the time-continuous system. The vertical dashed lines indicate the $B$ values of the stationary simulations used for the hotspot detection (red: bifurcation 1 in RM1 and RM2, orange: bifurcation 2 in RM1).

is between 0.1 and 0.96 (red area in Fig. 2). For RM2 we select the 8 grid cells in the south-west which show substantial collapse at $3.5 \mathrm{k}$ (purple area in Fig. 2).

Besides precipitation, growing degree days $\mathrm{GDD}_{0}$ are also a space and time dependent variable of the system which affects $V^{*}$ (Eq. 1). However, by choosing $\gamma=1.7 \times 10^{-5}$, the sensitivity of $V^{*}$ to changes in $\mathrm{GDD}_{0}$ is very small in the modified VECODE. Differentiation of $V^{*}$ with respect to $\delta$ as well as a graphical analysis reveals that shifts in $P$ direction do not exceed some $\mathrm{mm}$ for typical changes in $\delta$. As plants in arid regions are limited by water rather than temperature, the neglect of temperature fluctuations seems reasonable. Typical spatial differences in $\mathrm{GDD}_{0}$ (time means are between 7000 and 12000) exceed the temporal variability in North Africa (approx. 1000 at most grid cells) in PlaSim-VECODE-tr. Therefore, we prescribe a constant value of $\mathrm{GDD}_{0}$ (and thereby $\delta$ ) at each grid cell of our regression models. Each value corresponds to the average over all years and time slices $(9 \mathrm{k}-6 \mathrm{k}$ for RM1, $5.5 \mathrm{k}-3.6 \mathrm{k}$ for RM2). Hence, the function $V_{i}^{*}(P)$ very slightly depends on the particular grid cell $\mathrm{i}$, but is constant in time.

It remains to determine suitable parameter values of $P_{0_{i}}$, $s_{i}$, and $k_{i j}$ to reproduce the stability properties of PlaSimVECODE with the regression models. To this aim, we fit these parameters to our stationary PlaSim-VECODE-tr simulations using a multivariate linear regression:

First, we extend the vector $V_{i}$ at every year from PlaSimVECODE-tr by one additional dimension, assigned with the orbit year corresponding to each time slice. Although $V$ actually consists of trees and grass cover in VECODE, we can safely neglect this distinction, as tree cover is always close to 0 in the grid cells we consider. Using the extended vector as a predictor and the corresponding PlaSim-VECODE-tr time series of $P_{i}$ as responses, we calculate regression coefficients using the MATLAB function mvregress. Each $P_{0_{i}}$ is then obtained as the constant offset of the regression line, $s_{i}$ is its slope with regard to orbit year, and $k_{i j}$ are its slopes with regard to $V_{j}$ (Fig. 4).

\subsection{Robustness and stability properties of the regression models}

To investigate the stability properties of the two regression models over a range of $B$, we numerically determine deterministic equilibria and the eigenvalues of these equilibria as obtained from a linear stability analysis (Fig. 5). To obtain the eigenvalues, we derive the Jacobian of the corresponding time-continuous deterministic system (for which a bifurcation is indicated by an eigenvalue approaching 0 ) and 
calculate its properties by inserting the numerically obtained equilibrium. For the first value of $B$ (8.8 k for RM1, and $6 \mathrm{k}$ for RM2), we use $V_{i}=1$ as an initial condition and run the model to equilibrium. For all subsequent steps of $B$, we insert the previously obtained equilibrium as an initial condition (which always results in the same solution as using $V_{i}=1$ for any $B$ in our two regression models).

In both models the obtained equilibria are stable fixed points, as indicated by the negative real parts of all eigenvalues. Before a sudden transition to a different equilibrium occurs due to a saddle-node bifurcation, one eigenvalue approaches 0 . A reversed scanning of the $B$ range with our numeric approach to find equilibria indeed results in a static hysteresis (not shown). The equilibria coincide well with the green and desert equilibria found with PlaSim-VECODE-eq (Bathiany et al., 2012) which indicates that the regression model is of sufficient quality.

In RM1, there are several bifurcations along the forward branch, two in the $B$ range of interest: at approx. $8 \mathrm{k}$, grid cell 3 (marked in Fig. 2) collapses. At around $6.7 \mathrm{k}$, most other grid cells collapse in a second bifurcation.

This second bifurcation clearly corresponds to the disappearance of the green equilibrium in PlaSim-VECODE-eq (Bathiany et al., 2012). Considering that the variability which is still present to some extent in PlaSim-VECODE-eq prevents a detection of the green equilibrium close to the bifurcation point, the timing of the bifurcation also coincides well. However, the collapse of grid cell 3 at $8 \mathrm{k}$ only occurs in RM1, whereas in PlaSim-VECODE-eq vegetation cover is gradually reduced over time.

Figure 4 indicates that the relation between a certain $P_{i}$ and $V_{j}$ is generally rather weak. However, the large variations can partly be explained with the influence of the other 52 predictor variables which are not taken into account in Fig. 4. As the residuals of our regression are not Gaussian distributed and their variance depends on the predictors (heteroscedasticity), we refrain from calculations of errors or confidence intervals. Instead, we test the robustness of our results towards changes in the predictor variables: excluding certain time slices from PlaSim-VECODE-tr (e.g. 9k, $8.5 \mathrm{k}$ and $8 \mathrm{k}$ at the same time) and/or including the $5.5 \mathrm{k}$ experiment to determine the regression parameters for RM1 leads to similar results with regard to the system's stability properties. Also, slight changes in the selected grid cells to build RM1 (for example, excluding the rather stationary cells near the mediterranean and the 4 most northern grid cells) do not alter the properties of the regression model substantially. This even holds true if we replace the original PlaSim-VECODE time series by a set of 20000 bootstrapped pairs of $P$ and $V$ (Efron, 1979). However, some of these alternative regression models show additional bifurcations in RM1. Nonetheless, the main bifurcation point at which most elements of the system collapse in synchrony always occurs. The tendency of RM1 to show more bifurcations than PlaSim-VECODE may result from intrinsic limi- tations of our linear fit. For example, orbital forcing and its impact on annual precipitation does not change linearly over time.

In contrast, RM2 behaves more robustly as its 8 elements ( 80 coefficients) allow a more reliable regression than the 52 elements of RM1 (2808 coefficients). All elements in RM2 collapse in synchrony, regardless of the choice of time slices or the realisation in our bootstrapping experiments.

Altogether, the regression models therefore cannot reproduce the PlaSim-VECODE results in every aspect but qualitatively show many similarities and provide a simple and appropriate framework for EWS analysis.

The emergence of multiple equilibria from the noisy PlaSim-VECODE-tr time series provides further evidence that multiple deterministic equilibria are present in the modified PlaSim-VECODE but do not become apparent in probability density functions due to a noise-induced transition (Bathiany et al., 2012). Using an interactive noise level and an interactive vegetation timescale, as in Bathiany et al. (2012), leads to similar transitions as in PlaSim-VECODE-tr but in contrast to Bathiany et al. (2012) in a spatially explicit way (not shown).

\section{Hotspot detection in the regression models}

We can now answer the question from where each tipping in Fig. 5 originates by applying our hotspot detection algorithm:

1. We generate time slices of $100000 \mathrm{yr}$ each with RM1 and RM2. The chosen values of $B$ for these time slices are again depicted as dashed vertical lines in Fig. 5. Two time series are generated for each forcing, one with additive noise and one with multiplicative noise.

2. As the noise level is small, some grid cells in RM1 are already unvegetated and thus can be discarded as hotspot candidates (the desert cells in PlaSimVECODE-eq). We therefore do not consider grid cells where $V$ falls below 0.004 at any time step in any time slice.

3. To the rest of the grid cells, we apply the hotspot detection scheme presented in Part 1 of our study: in short, we repeatedly apply degenerate fingerprinting (Held and Kleinen, 2004) to a random selection of grid cells and each time determine an EWS. During this analysis we successively remove grid cells which contribute least to the signal and finally identify a hotspot from the resulting signal list.

The detection algorithm developed in Part 1 requires some parameters and options, in particular the definition of a signal (SD 1 or 2), the choice of an elimination rule (ER 1 or 2), the construction of the EOFs from covariance or correlation matrices and the use of autocorrelation or relative variance as 

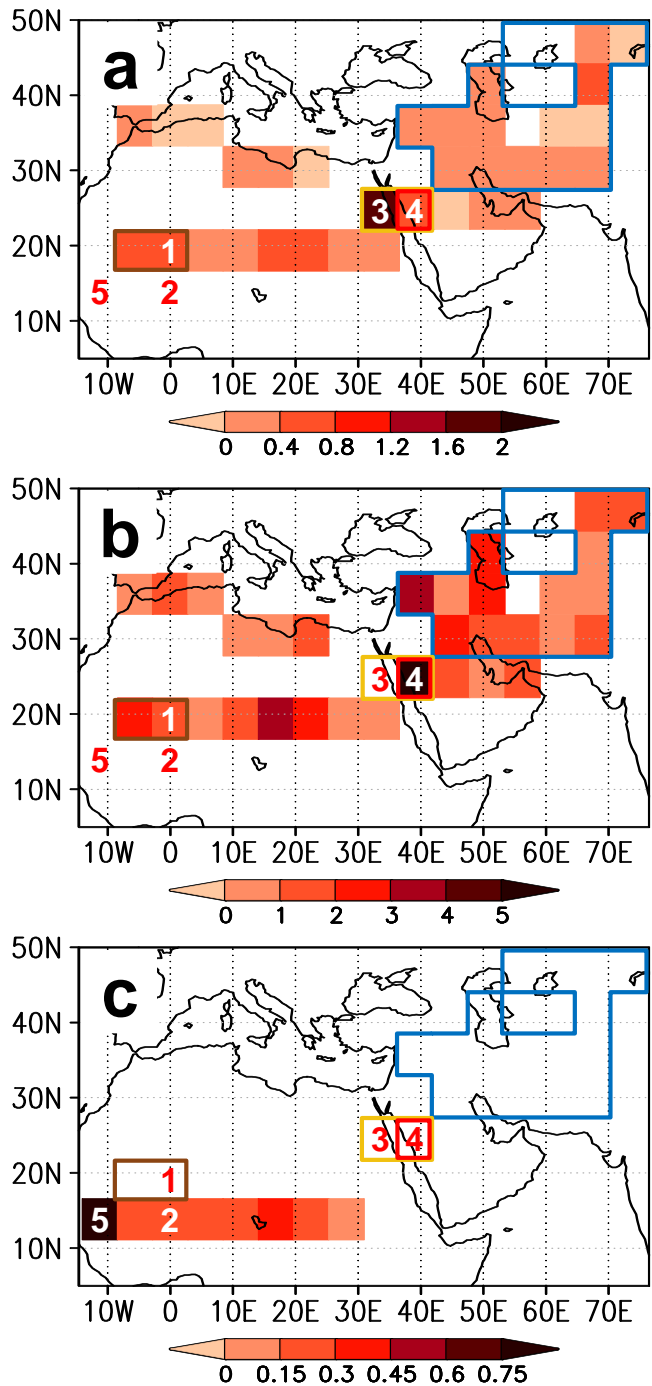

Fig. 6. Contribution of grid cells (weights) to the increasing autocorrelation as obtained with the hotspot detection algorithm, (a) in RM1; tipping point 1, (b) RM1; tipping point 2, (c) RM2. The noise in all time series is additive, hotspot detection is applied with $n_{\max }=3$, elimination rule 1 , and covariance-based EOFs. Numbers 1 to 5 denote the individual grid cells referred to in the text. The coloured areas are the areas of our perturbation experiments explained in Sect. 5.2.

an EWS (relative to the first value most distant from the tipping point). Here, we test all possible combinations of these options which are discussed in Part 1 of our article. To keep the algorithm sufficiently fast, the system under analysis is repeatedly divided into parts with a maximum number of elements prescribed by $n_{\max }$. Here, we use values of 3,5 and 8 for $n_{\max }$. For the successive removal of elements during the procedure, a relative threshold is applied which starts at an initial value $t_{\text {ini }}$ and is increased in steps of $t_{\text {inc }}$. For both parameters we use the standard values from Part 1: $t_{\text {ini }}=5 \%$ and $t_{\text {inc }}=5 \%$.
In order to illustrate the hotspots geographically, we indicate an element's weight at its corresponding grid cell (Fig. 6). As a weight we define the sum of signals a certain element contributes to, as illustrated by Table 3 in Part 1 of our article. It must be noted though that the random sampling and the systematic removal of elements during the hotspot detection algorithm only allows qualitative conclusions like the position of the hotspot. The quantitative differences between the grid cells in Fig. 6 should therefore not be overinterpreted. The significance of the coloured areas will be explained in Sect. 5.2

We find that before the collapse of grid cell 3 in RM1, this grid cell is detected as a hotspot of the transition. This is of course not surprising as this grid cell is the only one showing a collapse. In the more complex case of collapse 2 in RM1, its neighbouring grid cell 4 is identified as the hotspot of the transition. The collapse of the eight grid cells in RM2 is detected to be initiated by the most western grid cell (cell 5 ). Hence, each collapse in our regression models originates at one single grid cell.

To investigate the robustness of these results, we compare the results for all possible combinations of our parameter options as listed above (SD, ER, EOFs, EWS, $n_{\max }$ ). We find that the determined hotspots are always the same for all combinations of these parameter settings. Furthermore, omitting time slices or using bootstrapped versions of our regression models as explained in Sect. 3.2 yields the same hotspots despite the uncertainty in the regression parameters.

When reducing the length of the time series we generate with the regression models the hotspots clearly emerge from the noise until a total length of several $1000 \mathrm{yr}$ in case of autocorrelations and several $100-1000 \mathrm{yr}$ in case of variances. RM2 is even more robust: $100 \mathrm{yr}$ of each time series are sufficient to detect the hotspot when using relative variance as an EWS.

In summary, our detected hotspots are a very robust characteristic of PlaSim-VECODE. In the following section we document that they are also meaningful, in the sense that they yield information on the stability properties of PlaSimVECODE.

\section{Evaluation of results with PlaSim-VECODE}

To verify the detected hotspots we seek evidence for their existence in PlaSim-VECODE and an explanation in terms of the model's physics. As we apply PlaSim-VECODE with low resolution, present-day SSTs and a quite crude representation of physical surface parameters, the model cannot be expected to provide a very realistic climate of the mid-Holocene.

Despite these limitations, the large-scale features of the North African summer circulation are captured reasonably. We here focus on the conditions during July to September because in the model most precipitation in northern Africa and southwestern Asia occurs during these months. The 

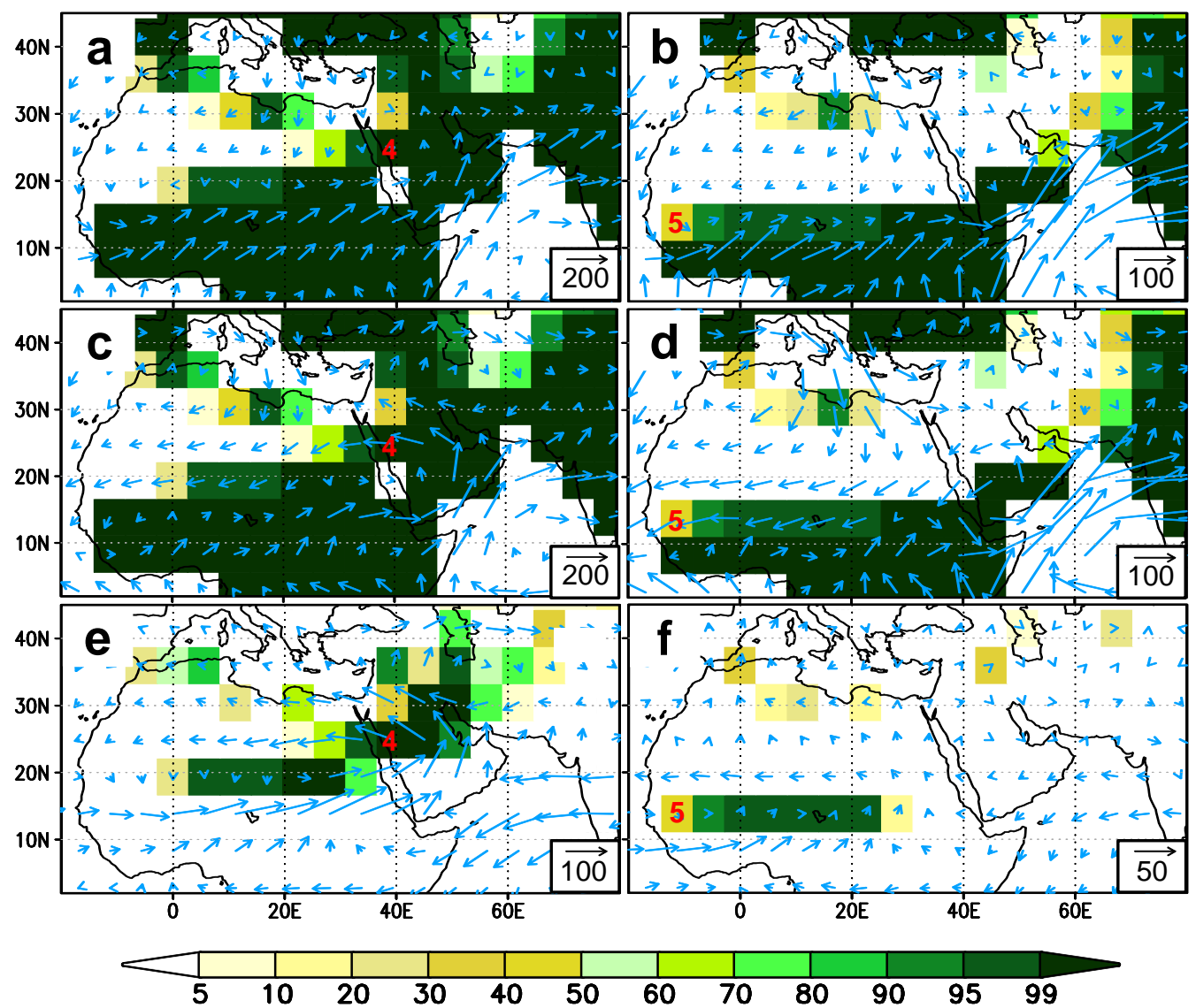

Fig. 7. Vertically integrated horizontal moisture fluxes (arrows) and vegetation cover (colours) in PlaSim-VECODE-eq. Left column (a, c, e): $8 \mathrm{k}$ conditions; right column (b, d, f): $4.5 \mathrm{k}$ conditions. (a)-(d): green equilibrium; (e)-(f): difference between green and desert equilibrium. (a)-(b): moisture fluxes are integrated over the two lowest atmosphere levels only; (c)-(f): moisture fluxes are integrated over the whole atmospheric column. Vegetation cover fractions in (a) and (c) as well as in (b) and (d) are the same. Fluxes are in $\mathrm{kg} /(\mathrm{ms})$, vegetation cover fractions in \%. Numbers 4 and 5 denote the individual grid cells referred to in the text.

south-westerly monsoon flow is confined to the lowest model levels and advects moisture over the North African continent towards the heat low in central northwestern Africa (Fig. 7a, b). The intertropical front is very prominently indicated by a surface convergence and a strong jump in specific moisture around $15-20^{\circ} \mathrm{N}$. To the north of this front, the north-easterly trades advect dry air from the Mediterranean region.

As in observations, easterly winds prevail in all tropospheric levels above the shallow monsoon flow. Due to the low model resolution, the African easterly jet (AEJ), tropical easterly jet (TEJ) and the low-level westerly jet (Patricola and Cook, 2007) cannot be captured well and the horizontal gradients in zonal wind are small. Since precipitation in the Sahel is related to the strength and position of these jets (Nicholson, 2009), the model cannot capture the small-scale nature of precipitation events. The seasonal migration of the rain belt and its northward shift during the mid-Holocene are nonetheless captured by PlaSim-VECODE. However, the zonal structure of the rainfall pattern is in conflict with observations. While the eastern Sahel is drier than the west in present-day observations (Andersson et al., 2010), precipitation in PlaSim-VECODE strongly increases towards the east. There, the south-westerly flow becomes even stronger and advects moisture from central Africa. This azonal structure is present in the complete Holocene.

\subsection{Collapse 2}

The west to east gradient in precipitation and the advection of moisture are also the key to understanding why the westernmost grid cell (grid cell 5) is a hotspot in RM2. The substantial precipitation gradient is reflected in the regression parameters $P_{0_{i}}$. In addition, the interaction matrix $\mathbf{k}$ reveals that the impact of grid cell 5 on other cells is exceedingly large for reasons explained below. When orbital forcing evolves, the precipitation pattern shifts towards the east. Therefore, grid cell 5 is the driest element in RM2. Its influence on its easterly neighbors due to moisture advection keeps the system green for a long time. When precipitation in cell 5 finally is too low for vegetation to be sustained, precipitation in the 


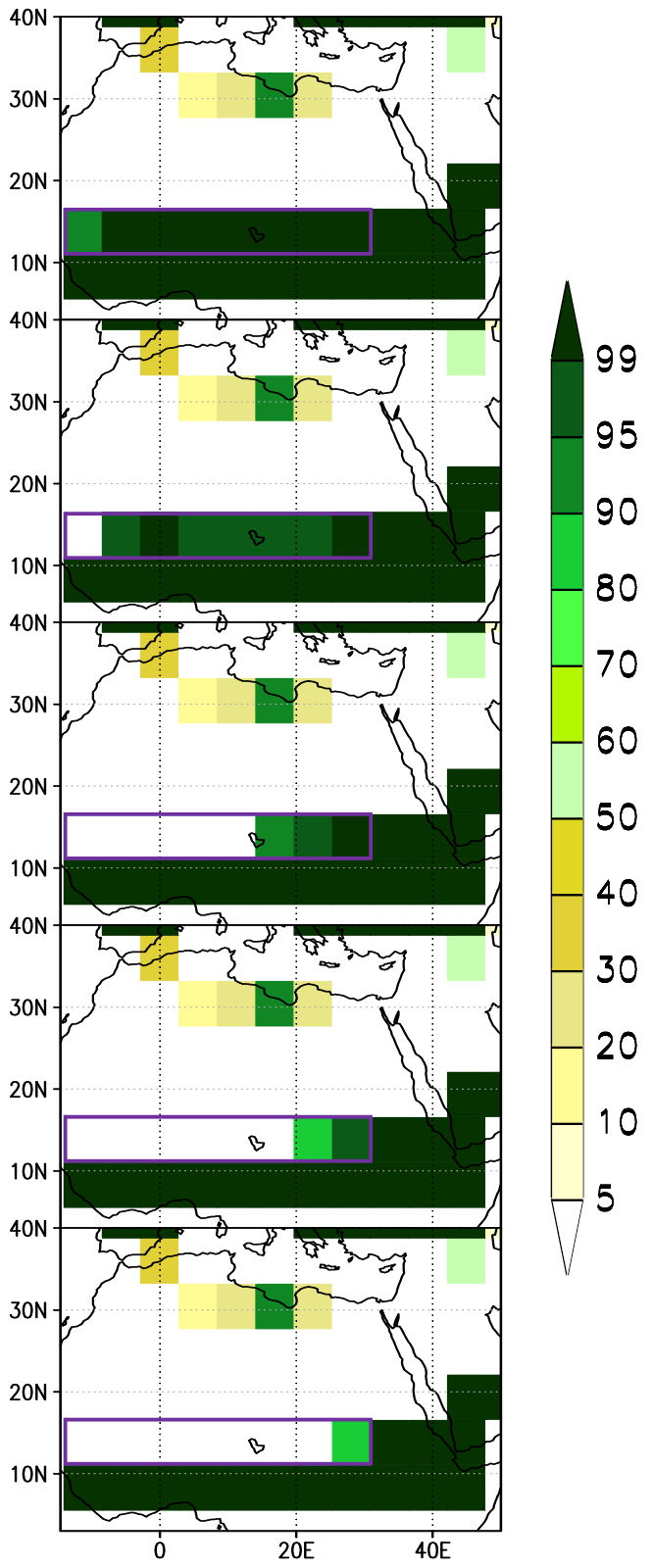

Fig. 8. The five equilibria of Eqs. (1), (2), and (3) for $4.5 \mathrm{k}$ conditions as obtained in Sect. 5.1. The regression involves the 8 grid cells enclosed in the purple box; cover fractions outside this area are set to mean conditions in PlaSim-VECODE-eq.

other cells also decreases below the critical threshold. Hence, these other elements experience an induced tipping and the hotspot is to be found at cell 5 .

The non-trivial structure of interactions $k_{i j}$ implies that more equilibria may exist in PlaSim-VECODE than those found by choosing global forest or desert initial conditions as in Bathiany et al. (2012). Our conceptual model framework (Eqs. 1, 2, and 3) is suitable to determine fixed points of the system in a more systematic way. To dispose of the deficiencies of including time in the regression model, we now apply the regression to our $4.5 \mathrm{k}$ simulation with PlaSimVECODE-tr only, which corresponds to dropping the term $s_{i} B$ in Eq. (2). Again, we consider the same line of eight grid cells as in RM2. As it is not possible to find the fixed points analytically, we randomy select 10 million initial conditions and run RM2 (without noise) to a steady state. As a strategy to sample the initial conditions in phase space we apply a regular, completely random, and a latin hypercube sampling (using MATLAB function lhsdesign). For every sampling method we obtain the same five deterministic solutions (Fig. 8). By reintroducing these fixed points as initial conditions in PlaSim-VECODE-eq with a coupling frequency of $30 \mathrm{yr}$ we can verify the existence of all five solutions in PlaSim-VECODE-eq.

The structure of these solutions is suggestive with regard to the position of the hotspot: all equilibria have in common that any green grid cell permits only green grid cells to its east. This feature is due to the advection of moisture with the westerly monsoon flow. In addition to this moisture recycling, the enhanced evaporation affects atmospheric stability and the circulation itself (Goessling and Reick, 2011). Also, an impact of easterly on westerly cells exists due to albedo induced changes in monsoon strength. In the case of grid cell 5 both effects work in the same direction which explains its large importance: first, it supplies additional moisture to its eastward neighbors via recycling. Second, it enhances the thermal low and thus the low-level south-westerly monsoon flow which supplies the more easterly region. As this flow is overcompensated by the export of moisture towards the west in higher levels, the vertically integrated moisture flux is towards the west (Fig. 7d), but the difference between green and desert equilibrium (Fig. 7f) indicates the enhancement of low-level westerlies due to the vegetation.

Thus, RM2 as shown in Fig. 5 stays in the greenest equilibrium for a long time, while some of the fixed points with intermediate vegetation cover disappear. When vegetation at the hotspot collapses, precipitation at all other grid cells becomes too low to sustain vegetation and the system drops into the driest equilibrium.

\subsection{Collapse 1}

The zonal gradient in precipitation and its shift over time are also present from $9 \mathrm{k}$ to $6 \mathrm{k}$. Like for RM2, the grid cells with the least precipitation are also at the western margin of the model region. This is the reason for the collapse of grid cell 3 in RM1, which has no consequences for the rest of the system. In contrast, our hotspot detection method identifies the rather wet grid cell 4 as the hotspot of collapse 1 (second collapse in RM1), implying a decreasing stability and thus an increasing sensitivity to perturbations at this point. To verify this result we initialise PlaSim-VECODE-eq with $8 \mathrm{k}$ forcing and a coupling frequency of $20 \mathrm{yr}$ in the green and desert equilibrium but impose a perturbation in certain test areas (enclosed by coloured boxes in Fig. 6). In case of the green 

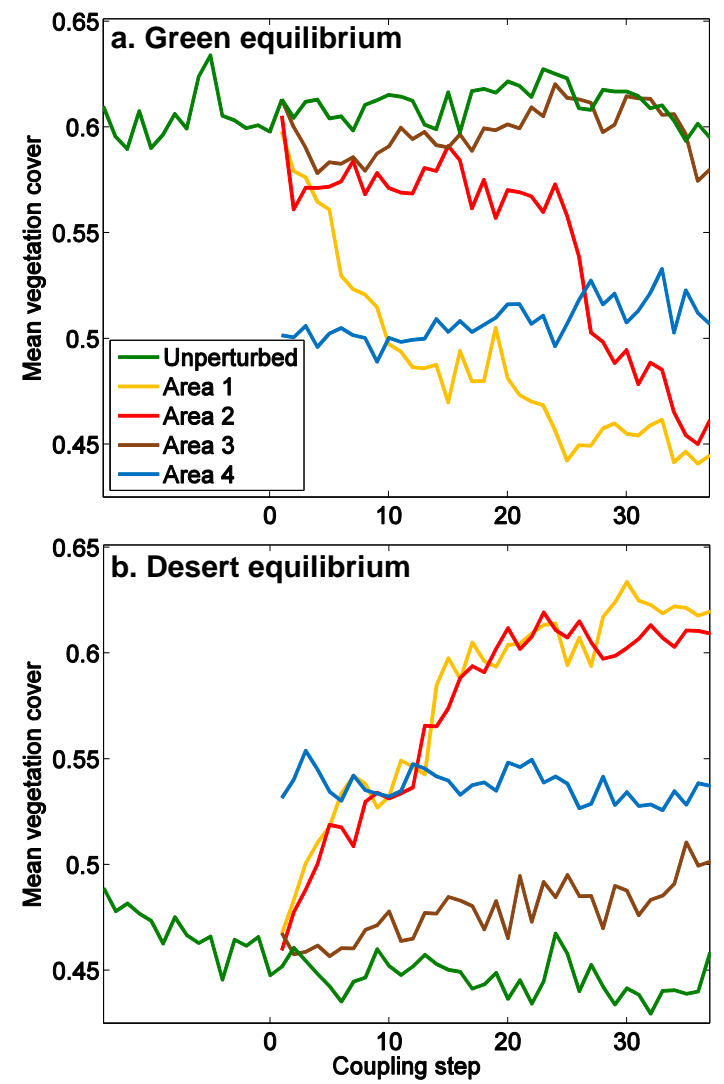

Fig. 9. Evolution of vegetation cover fraction in PlaSim-VECODEeq with perturbations in different areas. All vegetation cover fractions are averaged over the complete region shown in Figs. 10 and 6 $\left(5^{\circ} \mathrm{N}-50^{\circ} \mathrm{N}, 14.6^{\circ} \mathrm{W}-76.5^{\circ} \mathrm{E}\right)$. The colours correspond to the areas marked in Fig. 6, where vegetation cover is set to 0 (a) or 1 (b).

equilibrium, we set the test area to desert conditions, in case of the desert equilibrium we set it to $100 \%$ grass cover. In the test areas, cover fractions are kept fixed at these initial conditions, while the dynamic vegetation is still active in all other areas. As a result we find that the complete system can be forced to flip into the opposing equilibrium by a perturbation at grid cells 3 and 4 (area 1; Fig. 9). Even a perturbation in grid cell 4 only (area 2) has this effect, though after some time in an intermediate state in the case of green initial conditions. In contrast, the two westernmost grid cells in the Sahara (area 3) and even the complete north-eastern half of the model region (area 4) do not have a comparable effect on $V$ in the remaining system part, which remains unaffected by the perturbations (Fig. 10).

An analysis of the moisture fluxes at $8 \mathrm{k}$ reveals the reason for the model's vulnerability at the hotspot: North Africa, as well as Southwest Asia, are both supplied by moisture which originates in the Atlantic and Indian oceans and then passes over the Arabian peninsula (Fig. 7a, c). There the low-level circulation splits into an easterly part, turning back to North Africa, and a branch that extends northward over Southwest
Asia, and joins the mid-latitude westerlies. Therefore, the west African part of the bistable region not only receives moisture from the direct low-level monsoon flow but also from this moisturising of the easterlies aloft. The contribution of these two sources is most apparent in the difference between the green and desert equilibria in PlaSim-VECODEeq (Fig. 7e). With vegetation present, both sources are enhanced and contribute to the local convergence of moisture. The maximum surface pressure difference is located at the northern Red Sea, coinciding with the detected hotspot. Hence, imposing desert conditions in this key area weakens the heat low and the cyclonic circulation over Arabia and thus cuts off the moisture supply to both circulation branches. Therefore, the rest of the vegetation disappears and the resulting lack of moisture convergence leads to a rapid transition to the desert equilibrium in PlaSim-VECODE.

\section{Conclusions}

The possibility to use indicators of slowing down to analyse the climate system has been documented extensively in recent years (Held and Kleinen, 2004; Dakos et al., 2008; Lenton et al., 2009, 2012). In Part 1 of this two-part paper we have proposed a new method to infer the position of hotspots in a diagnostic way from model output. Here, we have applied our method to a regression model based on results from a global atmosphere-vegetation model, and have identified its hotspots.

We have thus documented that the hotspot detection method can provide information on the causality of a tipping and on the sensitivity of the model under consideration. If the model represents reality in an adequate way, an analysis with EWS can indicate where the earth system is particularly vulnerable to perturbations. On the other hand, if the model behaves in an unrealistic way, a hotspot detection analysis may improve the knowledge on its shortcomings and make its limitations more apparent. This knowledge can be beneficial for further model development. In case of PlaSimVECODE, a perturbation of surface parameters at a single grid cell can change the circulation on a regional to continental scale, and it remains doubtful if this result is realistic. However, we have identified the Red Sea area in the model as a crucial region for the moisture supply of Northern Africa and Southwest Asia at $8 \mathrm{k}$.

The application of EWS to infer this information was only possible via the somewhat technical detour of fitting a regression model to PlaSim-VECODE-tr. A direct analysis of the model output would have yielded inscrutable results as the requirements for EWS are not met. This restriction illustrates that applying EWS-based tools of analysis to data of unknown origin is problematic. Instead, it should always be established if the conceptual framework of analysis is an adequate description of the processes which have generated the data. In the case of PlaSim-VECODE-tr, we have 


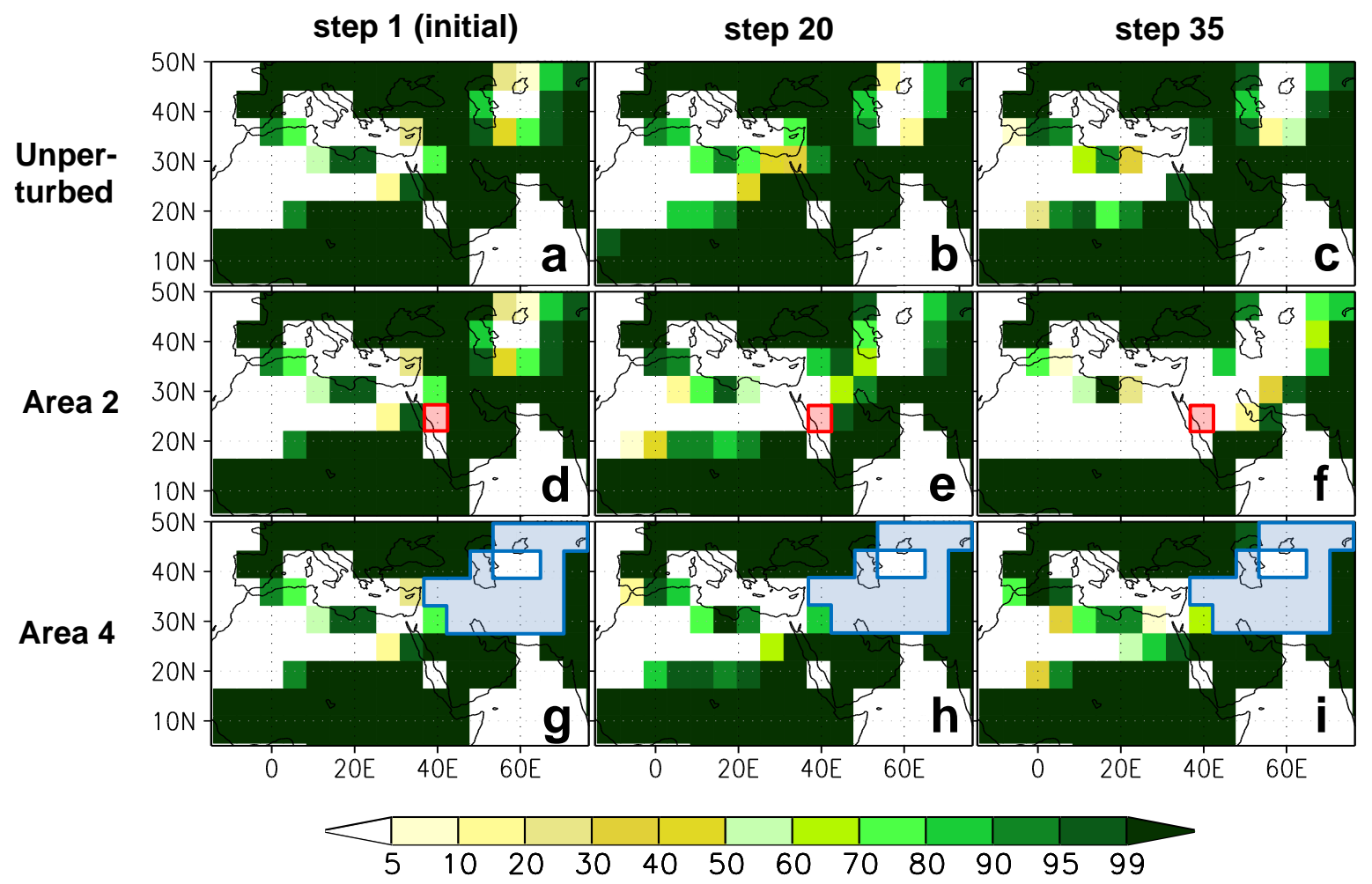

Fig. 10. Vegetation cover fractions (in \%) in asynchronously coupled PlaSim-VECODE after initialisation in the greenest equilibrium with no perturbation $(\mathbf{a}-\mathbf{c})$, no vegetation in area 2 (red, $\mathbf{d}-\mathbf{f})$, and no vegetation in area 4 (blue, $\mathbf{g}-\mathbf{i})$.

documented before that the large multiplicative noise is in conflict with this concept (Bathiany et al., 2012). In particular, the small noise approximation breaks down under such conditions: higher-order terms would become important so that the linearisation around an equilibrium and thus the link between EWS and local stability would not be strictly valid anymore. Even more importantly, the multiplicative nature of the noise leads to a noise-induced transition. The tipping point then depends on properties of the noise and does not coincide with the deterministic bifurcation point. Variance would be a particularly unreliable indicator under such conditions. If it depends on the forcing (directly or indirectly through other variables), variance can decrease towards the tipping point if these effects overcompensate the influence of slowing down (Dakos et al., 2012).

Although the green equilibria in PlaSim-VECODE-eq disappear due to an instability at the corresponding hotspot, we therefore cannot draw a conclusion regarding the causality of the collapses in PlaSim-VECODE-tr. There, the large variability eliminates the complex deterministic stability properties and the hotspots of the model are probably much less focussed.

The application of a hotspot detection scheme to other (potentially more complex) models therefore requires a thorough mechanistic understanding. First, it must be established that a sudden transition results from a destabilisation of an equilibrium due to internal feedbacks when the forcing is varied. This concept is in contrast to other possible reasons for sudden changes such as a discontinuous response function (not involving feedbacks) or chaotic dynamics like regime changes or intermittency that do not require any external parameter changes. The hotspot detection scheme therefore cannot dispose of the task to determine the most appropriate minimal model for explaining a sudden transition.

Second, the relation between the stability of an equilibrium and EWS is not a priori clear in a complex model. It must therefore be understood how the variability arises and how it affects the variable under consideration.

Third, the critical subsystem that is supposed to show slowing down must be identified so that an appropriate variable is chosen in the analysis.

The challenge for applying the hotspot detection scheme is therefore an investigative and intellectual challenge rather than a technical one. If the conditions for the applicability of EWS are met, the hotspot detection scheme is easy to apply as it is only a diagnostic tool and no changes to the model under analysis are required.

However, one technical limitation of the hotspot detection method is the requirement of very long time series, a condition hardly to be fulfilled by complex earth system models. In example system 3 in Part 1 of our study, we needed time series of the order of 10000-100 $000 \mathrm{yr}$ (with a relaxation time 
of $5 \mathrm{yr}$ ) to time steps (the dynamic system's relaxation time being 5) to obtain robust results. In the case of our regression models the results are much more promising. Even in RM1 with its 52 state variables, the hotspot is detectable from several 100 to 1000 time steps, and is basically independent of parameter choices during the analysis. The reason is that the hotspots consist of one single element which is well separable from the others, in contrast to our idealised setting of 9 identical elements in Part 1.

Models with higher spatial resolution could therefore pose a more difficult challenge if hotspots consist of many grid cells whose individual signal is hard to distinguish from others. The demand of long time series to increase the significance of the results would be particularly problematic regarding the computing time for such higher resolution models. However, large hotspots can still be detected if the system is divided into larger parts (determined by parameter $n_{\max }$ ) which would slow down the hotspot detection algorithm. As the increase in computing time of the algorithm results from the large number of possible combinations of elements that are considered independently, parallel computing could be applied to speed up the hotspot detection algorithm to some extent.

This issue relates to the problem of finding multiple steady states in the sense that they are difficult to identify in complex models. Due to the vast number of variables in a global climate model, strategies like hysteresis experiments or choosing different initial conditions are no fail-proof methods. It can be speculated that this caveat is one reason why multiple steady states have not been found in complex climate models in contrast to low-dimensional models. The detection of different stable equilibria in PlaSim-VECODE turned out to be possible using our regression model. However, the applicability of such an approach is very limited: (1) in PlaSimVECODE, the variability is large enough to sufficiently sample large parts of the phase space. (2) The regression can only be done in a limited area or for low resolution, otherwise too many regression coefficients would need to be estimated. (3) We based our regression model on the knowledge of $V^{*}(P)$. In case of a more complex vegetation model, many more variables would be involved and the relationships would be less clear.

Despite all these structural limitations, our method is generic in the sense that it is independent of the physical meaning of the model. For example, it may be applied to fluctuations in sea ice cover close to the snowball earth bifurcation (Lucarini et al., 2010; Voigt and Marotzke, 2010).

It therefore seems possible that the hotspot detection method or related approaches can yield useful information on the susceptibility not only of climate models but also of other systems.
Acknowledgements. S.B. is grateful to Thomas Kleinen for his helpful comments, to Holger Kantz and his group for their kind advice, to Steven Lade for the fruitful discussion and to Robert Schoetter for exciting bets at the edge of moral integrity. K.F., Max Planck Fellow, acknowledges the support by the Max Planck Society. This work is funded by Deutsche Forschungsgesellschaft, Cluster of Excellence "CliSAP" (DFG EXC 177). The simulations were performed at the German Climate Computing Center (DKRZ).

The service charges for this open access publication have been covered by the Max Planck Society.

Edited by: A. Kleidon

\section{References}

Andersson, A., Bakan, S., and Grassl, H.: Satellite derived precipitation and freshwater flux variability and its dependence on the North Atlantic Oscillation, Tellus A, 62, 453-468, doi:10.1111/j.1600-0870.2010.00458.x, 2010.

Bathiany, S., Claussen, M., and Fraedrich, K.: Implications of climate variability for the detection of multiple equilibria and for rapid transitions in the atmosphere-vegetation system, Clim. Dynam., 38, 1775-1790, doi:10.1007/s00382-011-1037-x, 2012.

Bathiany, S., Claussen, M., and Fraedrich, K.: Detecting hotspots of atmosphere-vegetation interaction via slowing down - Part I: A stochastic approach, Earth Syst. Dynam., 4, 63-78, doi:10.5194/esd-4-63-2013, 2013.

Brovkin, V., Ganopolski, A., and Svirezhev, Y.: A continuous climate-vegetation classification for use in climate-biosphere studies, Ecol. Model., 101, 251-261, doi:10.1016/S03043800(97)00049-5, 1997.

Brovkin, V., Claussen, M., Petoukhov, V., and Ganopolski, A.: On the stability of the atmosphere-vegetation system in the Sahara/Sahel region, J. Geophys. Res.-Atmos., 103, 31613-31624, doi:10.1029/1998JD200006, 1998.

Brovkin, V., Bendtsen, J., Claussen, M., Ganopolski, A., Kubatzki, C., Petoukhov, V., and Andreev, A.: Carbon cycle, vegetation, and climate dynamics in the Holocene: experiments with the CLIMBER-2 Model, Global Biogeochem. Cy., 16, 1139, doi:10.1029/2001GB001662, 2002.

Carpenter, S. R. and Brock, W. A.: Rising variance: a leading indicator of ecological transition, Ecol. Lett., 9, 308-315, doi:10.1111/j.1461-0248.2005.00877.x, 2006.

Carpenter, S. R., Cole, J. J., Pace, M. L., Batt, R., Brock, W. A., Cline, T., Coloso, J., Hodgson, J. R., Kitchell, J. F., Seekell, D. A., Smith, L., and Weidel, B.: Early warnings of regime shifts: a whole-ecosystem experiment, Science, 332, 1079-1082, doi:10.1126/science.1203672, 2011.

Claussen, M.: On coupling global biome models with climate models, Clim. Res., 4, 203-221, doi:10.3354/cr004203, 1994.

Claussen, M.: Modeling bio-geophysical feedback in the African and Indian monsoon region, Clim. Dynam., 13, 247-257, doi:10.1007/s003820050164, 1997.

Claussen, M.: On multiple solutions of the atmosphere-vegetation system in present-day climate, Global Change Biol., 4, 549-559, doi:10.1046/j.1365-2486.1998.t01-1-00122.x, 1998.

Claussen, M., Kubatzki, C., Brovkin, V., Ganopolski, A., Hoelzmann, P., and Pachur, H. J.: Simulation of an abrupt change in 
Saharan vegetation in the mid-Holocene, Geophys. Res. Lett., 26, 2037-2040, doi:10.1029/1999GL900494, 1999.

Collins, M. R. and Teh, H. C.: Neutron-scattering observations of critical slowing down of an Ising system, Phys. Rev. Lett., 30, 781-784, doi:10.1103/PhysRevLett.30.781, 1973.

Contamin, R. and Ellison, A. M.: Indicators of regime shifts in ecological systems: What do we need to know and when do we need to know it?, Ecol. Appl., 19, 799-816, doi:10.1890/08-0109.1, 2009.

Dakos, V., Scheffer, M., van Nes, E. H., Brovkin, V., Petoukhov, V., and Held, H.: Slowing down as an early warning signal for abrupt climate change, P. Natl. Acad. Sci. USA, 105, 1430814312, doi:10.1073/pnas.0802430105, 2008.

Dakos, V., van Nes, E. H., Donangelo, R., Fort, H., and Scheffer, M.: Spatial correlation as leading indicator of catastrophic shifts, Theor. Ecol., 3, 163-174, doi:10.1007/s12080-009-00606, 2010.

Dakos, V., Kefi, S., Rietkerk, M., van Nes, E. H., and Scheffer, M.: Slowing down in spatially patterned ecosystems at the brink of collapse, Am. Nat., 177, E153-E166, doi:10.1086/659945, 2011.

Dakos, V., van Nes, E. H., D’Odorico, P., and Scheffer, M.: Robustness of variance and autocorrelation as indicators of critical slowing down, Ecology, 93, 264-271, doi:10.1890/11-0889.1, 2012.

deMenocal, P., Ortiz, J., Guilderson, T., Adkins, J., Sarnthein, M., Baker, L., and Yarusinsky, M.: Abrupt onset and termination of the African Humid Period: rapid climate responses to gradual insolation forcing, Quaternary Sci. Rev., 19, 347-361, doi:10.1016/S0277-3791(99)00081-5, 2000.

Ditlevsen, P. D. and Johnsen, S. J.: Tipping points: early warning and wishful thinking, Geophys. Res. Lett., 37, L19703, doi:10.1029/2010GL044486, 2010.

Donangelo, R., Fort, H., Dakos, V., Scheffer, M., and Van Nes, E. H.: Early warnings for catastrophic shifts in ecosystems: comparison between spatial and temporal indicators, Int. J. Bifurcat. Chaos, 20, 315-321, doi:10.1142/S0218127410025764, 2010.

Drake, J. M. and Griffen, B. D.: Early warning signals of extinction in deteriorating environments, Nature, 467, 456-459, doi:10.1038/nature09389, 2010.

Efron, B.: 1977 Rietz Lecture - Bootstrap Methods Another Look At the Jackknife, Ann. Stat., 7, 1-26, doi:10.1214/aos/1176344552, 1979.

Fraedrich, K.: Catastrophes and resilience of a zero-dimensional climate system with ice-albedo and greenhouse feedback, Q. J. Roy. Meteor. Soc., 105, 147-167, doi:10.1002/qj.49710544310, 1979.

Fraedrich, K.: A suite of user-friendly global climate models: hysteresis experiments, Eur. Phys. J. Plus., 127, 53, doi:10.1140/epjp/i2012-12053-7, 2012.

Fraedrich, K., Jansen, H., Kirk, E., Luksch, U., and Lunkeit, F.: The Planet Simulator: towards a user friendly model, Meteorol. Z., 14, 299-304, doi:10.1127/0941-2948/2005/0043, 2005.

Goessling, H. F. and Reick, C. H.: What do moisture recycling estimates tell us? Exploring the extreme case of nonevaporating continents, Hydrol. Earth Syst. Sc., 15, 3217-3235, doi:10.5194/hess-15-3217-2011, 2011.

Guttal, V. and Jayaprakash, C.: Changing skewness: an early warning signal of regime shifts in ecosystems, Ecol. Lett., 11, 450460, doi:10.1111/j.1461-0248.2008.01160.x, 2008.

Guttal, V. and Jayaprakash, C.: Spatial variance and spatial skewness: leading indicators of regime shifts in spatial ecological systems, Theor. Ecol., 2, 3-12, doi:10.1007/s12080-008-0033-1, 2009.

Held, H. and Kleinen, T.: Detection of climate system bifurcations by degenerate fingerprinting, Geophys. Res. Lett., 31, L23207, doi:10.1029/2004GL020972, 2004.

Horsthemke, W. and Lefever, R.: Noise-Induced Transitions, Springer, 1984.

Irizarry-Ortiz, M. M., Wang, G. L., and Eltahir, E. A. B.: Role of the biosphere in the mid-Holocene climate of West Africa, J. Geophys. Res.-Atmos., 108, 4042, doi:10.1029/2001JD000989, 2003.

Kleinen, T., Held, H., and Petschel-Held, G.: The potential role of spectral properties in detecting thresholds in the Earth system: application to the thermohaline circulation, Ocean Dynam., 53, 53-63, doi:10.1007/s10236-002-0023-6, 2003.

Lenton, T. M.: Early warning of climate tipping points, Nature Clim. Change, 1, 201-209, doi:10.1038/NCLIMATE1143, 2011.

Lenton, T. M., Myerscough, R. J., Marsh, R., Livina, V. N., Price, A. R., and Cox, S. J.: Using GENIE to study a tipping point in the climate system, Phil. Trans. R. Soc. A, 367, 871-884, doi:10.1098/rsta.2008.0171, 2009.

Lenton, T. M., Livina, V. N., Dakos, V., van Nes, E. H., and Scheffer, M.: Early warning of climate tipping points from critical slowing down: comparing methods to improve robustness, Phil. Trans. R. Soc. A, 370, 1185-1204, doi:10.1098/rsta.2011.0304, 2012.

Liu, Z. Y., Wang, Y., Gallimore, R., Notaro, M., and Prentice, I. C.: On the cause of abrupt vegetation collapse in North Africa during the Holocene: climate variability vs. vegetation feedback, Geophys. Res. Lett., 33, L22709, doi:10.1029/2006GL028062, 2006.

Livina, V. N. and Lenton, T. M.: A modified method for detecting incipient bifurcations in a dynamical system, Geophys. Res. Lett., 34, L03712, doi:10.1029/2006GL028672, 2007.

Lucarini, V., Fraedrich, K., and Lunkeit, F.: Thermodynamic analysis of snowball earth hysteresis experiment: efficiency, entropy production and irreversibility, Q. J. Roy. Meteor. Soc., 136, 2-11, doi:10.1002/qj.543, 2010.

Nicholson, S. E.: A revised picture of the structure of the "monsoon" and land ITCZ over West Africa, Clim. Dynam., 32, 11551171, doi:10.1007/s00382-008-0514-3, 2009.

Oborny, B., Meszena, G., and Szabo, G.: Dynamics of populations on the verge of extinction, Oikos, 109, 291-296, doi:10.1111/j.0030-1299.2005.13783.x, 2005.

Patricola, C. M. and Cook, K. H.: Dynamics of the West African monsoon under mid-Holocene precessional forcing: regional climate model simulations, J. Climate, 20, 694-716, doi:10.1175/JCLI4013.1, 2007.

Renssen, H., Brovkin, V., Fichefet, T., and Goosse, H.: Holocene climate instability during the termination of the African Humid Period, Geophys. Res. Lett., 30, 1184, doi:10.1029/2002GL016636, 2003.

Scheffer, M., Carpenter, S., Foley, J. A., Folke, C., and Walker, B.: Catastrophic shifts in ecosystems, Nature, 413, 591-596, doi:10.1038/35098000, 2001.

Scheffer, M., Bascompte, J., Brock, W. A., Brovkin, V., Carpenter, S. R., Dakos, V., Held, H., van Nes, E. H., Rietkerk, M., and Sugihara, G.: Early-warning signals for critical transitions, Nature, 461, 53-59, doi:10.1038/nature08227, 2009.

Thompson, J. M. T. and Sieber, J.: Climate tipping as a noisy bifurcation: a predictive technique, IMA J. Appl. Math., 76, 27-46, 
doi:10.1093/imamat/hxq060, 2011a.

Thompson, J. M. T. and Sieber, J.: Predicting climate tipping as a noisy bifurcation: a review, Int. J. Bifurcat. Chaos, 21, 399-423, doi:10.1142/S0218127411028519, 2011 b.

van Nes, E. H. and Scheffer, M.: Slow recovery from perturbations as a generic indicator of a nearby catastrophic shift, Am. Nat., 169, 738-747, doi:10.1086/516845, 2007.

Veraart, A. J., Faassen, E. J., Dakos, V., van Nes, E. H., Lurling, M., and Scheffer, M.: Recovery rates reflect distance to a tipping point in a living system, Nature, 481, 357-359, doi:10.1038/nature10723, 2012.

Voigt, A. and Marotzke, J.: The transition from the present-day climate to a modern Snowball Earth, Clim. Dynam., 35, 887-905, doi:10.1007/s00382-009-0633-5, 2010.

Wang, G. L.: A conceptual modeling study on biosphereatmosphere interactions and its implications for physically based climate modeling, J. Climate, 17, 2572-2583, doi:10.1175/15200442(2004)017<2572:ACMSOB > 2.0.CO;2, 2004.
Wang, G. L. and Eltahir, E. A. B.: Biosphere-atmosphere interactions over West Africa. II: Multiple climate equilibria, Q. J. Roy. Meteor. Soc., 126, 1261-1280, doi:10.1002/qj.49712656504, 2000.

Wissel, C.: A universal law of the characteristic return time near thresholds, Oecologia, 65, 101-107, doi:10.1007/BF00384470, 1984.

Wolff, U.: Critical slowing down, Nucl. Phys. B-Proc. Sup., 17, 93102, doi:10.1016/0920-5632(90)90224-I, 1990.

Zeng, N. and Neelin, J. D.: The role of vegetation-climate interaction and interannual variability in shaping the African savanna, J. Climate, 13, 2665-2670, doi:10.1175/15200442(2000)013<2665:TROVCI >2.0.CO;2, 2000. 